\title{
Evaluación Energética del Sistema de Calentamiento en una Refinería de Aceites Usados de Motor
}

\author{
Irina León González, $\mathrm{MSc}^{1}$, Yris González, $\mathrm{PhD}^{1}$, Sergio A. Pérez, $\mathrm{PhD}^{2}$, Alexander Espinoza, Ing. ${ }^{1}$, \\ Simón Macías, Ing. ${ }^{1}$ \\ ${ }^{1}$ Escuela Superior Politécnica del Litoral,Ecuador, iribleon@espol.edu.ec, ykgonzal@espol.edu.ec, \\ apespino@espol.edu.ec, samacias@espol.edu.ec \\ ${ }^{2}$ Universidad de Carabobo,Venezuela, sperez@uc.edu.ve
}

\begin{abstract}
Resumen: Este trabajo presenta el estudio del análisis energético en el circuito de aceite térmico de una planta de re-refinación de aceite usado de motor, utilizando el software PRO/II®. Esta herramienta toma datos del proceso real y módulos de cálculo que modelan equipos de proceso, y al simular el funcionamiento del equipo que lo componen, es capaz de proporcionar información sobre datos energéticos y másicos, que ayudarán a verificar en qué puntos del proceso es importante aprovechar la energía y evaluar a través de un balance energético el potencial energético del sistema térmico evaluado. El estudio realizado en una refinería en operación, con una capacidad de procesamiento de $1088.64 \mathrm{~L} / \mathrm{h}$ de aceite usado de motor, indica que, en la caldera para calentamiento de aceite térmico, presenta una eficiencia del $63.2 \%$, respecto al total de energía generada por efectos de una combustión total y completa de diésel. Igualmente se determinó una pérdida de energía al ambiente en la caldera de un $8.33 \%$, y se tiene una disponibilidad de recuperación de energía, proveniente de los gases calientes de un $28.47 \%$.

Palabras Claves: Simulación, Análisis energético, balance de energía, potencial energético.
\end{abstract}

Abstract- This paper describes the study of the energy analysis of the system thermal oil for a used motor oil rerefining plant, using PRO/II® software. This tool takes data from a real process and units modeling the equipment to simulating the operation of the equipment that makes up the process, it is capable of providing information on energy and mass data that will help to verify at which points in the process it is important to take advantage of energy and evaluate through an energy balance the energy potential of the evaluated thermal system. The study carried out in a refinery in operation, with a processing capacity of 1088.64 $L / h$ of used motor oil, indicates that, in the boiler for heating thermal oil, it has an efficiency of $63.2 \%$, concerning to the total energy generated by the effects of a total and complete combustion of diesel. Likewise, an energy loss to the environment in the boiler of $8.33 \%$ was determined, and

Digital Object Identifier (DOI):

http://dx.doi.org/10.18687/LEIRD2021.1.1.12

ISSN: 2414-6390 ISBN: 978-958-52071-9-6 there is an availability of energy recovery, from hot gases of $28.47 \%$.

Keywords: simulation, energy analysis, energy balance, potential energy.

\section{INTRODUCCIÓN}

Los aceites lubricantes son productos químicos con una composición del 80 al $90 \%$ de destilados del petróleo y del 20 al $10 \%$ de aditivos, respectivamente. Además, estos aceites contribuyen en la disminución de la fricción, corrosión y actúa como medio de transferencia de calor en varios equipos. En el transcurso de su ciclo de operación, los aceites lubricantes se debilitan y degradan en sus propiedades, tales como: punto de inflamación, gravedad específica, viscosidad y entre otros. Estos cambios en sus propiedades y la presencia de contaminantes (agua, cenizas, barnices, etc.) convierten al aceite lubricante en inadecuado para su uso [1].

Los aceites lubricantes usados se consideran como residuos peligrosos debido a la presencia de metales pesados (arsenio, cromo, cadmio y plomo) y compuestos químicos, tales como, hidrocarburos aromáticos policíclicos, benceno, solventes clorados, entre otros. En el manejo de estos residuos se debe evitar su combustión, debido a que contaminan el aire y provocan enfermedades cancerígenas en las personas.

Hoy en día, se han incorporado nuevas tecnologías para la re-refinación de los aceites lubricantes usados e incluso se destaca que los nuevos procesos ofrecen mejor rendimiento en comparación a los procesos convencionales. Además, la regeneración de los aceites lubricantes usados promueve una disminución del 90\% de los impactos ambientales [2].

Existen diferentes tecnologías para la regeneración de la base lubricante a partir de los aceites usados, tales como, ácido-arcilla; destilación al vacío; evaporación de película delgada, extracción por solventes e hidro-tratamiento. La selección de la tecnología de tratamiento debe considerar los siguientes criterios: seguridad, salud, sostenibilidad y costos.

El tratamiento de los residuos de aceites usados promueve la economía circular debido a que reducen la 
contaminación mientras promueven los beneficios sociales. Por ejemplo, un estudio en Ecuador indica que, si se tratará 7330 ton por año de aceite usado en la ciudad de Cuenca, se tuviera un ahorro económico en el país de \$30 millones al año al reducir la importación de aceite lubricante [3].

El uso de los simuladores ha tenido un gran impacto en los procesos industriales, utilizados para evaluar el diseño de los equipos, su eficiencia e impacto ambiental. En el caso de procesos de reciclado de aceites lubricantes, se evidencian dos estudios realizados en ASPEN. Un primer estudio, para la evaluación ambiental y energético del proceso de rerefinación de los residuos de aceite con propano y una serie de destilaciones. En este estudio se determinó que este tipo de proceso genera menor impacto ambiental que la refinación convencional de aceite virgen [4]. Un segundo estudio, donde se evalúa la factibilidad de un proceso de re-refinación con un solvente ternario, determinándose una alta rentabilidad del proceso [5].

La evaluación energética busca reducir los costos energéticos de los equipos, utilizando nuevas tecnologías o aprovechando corrientes del proceso [6]. Los resultados de las evaluaciones energéticas ofrecen las siguientes ventajas: una disminución del $15 \%$ de consumo eléctrico y un ahorro de consumo de combustible entre el $8-15 \%$ por producto obtenido [7]. En 2021, un modelo de refinación (MESSAGEix-petróleo) para determinar el consumo de energía y las emisiones en el proceso, y los resultados demostraron que las refinerías en China podrían reducir el consumo de energía y emisiones de $\mathrm{CO}_{\mathrm{x}}$ en un $12 \%$ y $10 \%$ respectivamente [8].

El objetivo principal de esta investigación es realizar la evaluación energética del circuito de aceite térmico de un proceso de re-refinación de aceites usados a las condiciones actuales de operación, para cuantificar el uso actual de la energía en las diferentes secciones, y la evaluación de potencial energético recuperable.

Para la investigación, la información fue obtenida en una empresa dedicada a la re-refinación de aceites usado de motor, ubicada en la ciudad de Guayaquil, Ecuador, donde se procesa actualmente aproximadamente un flujo de alimentación de $1088.64 \mathrm{~L} / \mathrm{h}$ de aceite usado, produciendo $687.96 \mathrm{~L} / \mathrm{h}$ de base lubricante [9]. La tecnología del proceso está soportada en la patente de Jerome Nichols y Tyler Humphreys [10], que propone una serie de destilaciones simples al vacío, y los balances energético y masico se obtuvieron a partir de la simulación con programa $\boldsymbol{P R O / I I}{ }^{\circledR}$.

\section{MATERIALES Y MÉTODOS}

\section{A. Descripción del Proceso}

El proceso de refinación de aceites usados está conformado por tres etapas denominadas SKID 1, SKID2 y SKID 3; representan al conjunto de equipos conformados por intercambiador de calor, destilador flash, condensador, tanque de recolección de condensado, bomba de vacío y bombas para alimentación y salida de aceite usado.

Adicionalmente se dispone de un circuito de aceite térmico, constituido principalmente por una caldera a diésel, y red de distribución del aceite caliente, para suministrar la energía requerida en las etapas de evaporación del proceso de refinación,

En la Fig. 1 se presenta el diagrama de flujo del proceso con la respectiva identificación de cada equipo. El proceso inicia con la alimentación del aceite usado, mediante la bomba P-101 hacia la etapa SKID 1, donde el primer separador V-101 opera a una presión de vacío menor a 13.79 KPa y una temperatura determinada en el intercambiador HE101. En esa separación inicial se remueve el agua presente en la corriente de aceite usado, además de las fracciones de hidrocarburos ligeros, que son luego condensadas en el intercambiador HE-105.

Después de la primera separación, el aceite es enviado por la bomba P-102 hacia el separador flash V-102, se calienta previamente a través del intercambiador de calor HE102 a una temperatura superior a $260{ }^{\circ} \mathrm{C}$, con el fin de eliminar en esta etapa el diésel en el destilador flash, a una presión de vacío inferior a $13.79 \mathrm{KPa}$, los vapores son condensados por medio del intercambiador de calor HE-106.

Finalmente, la corriente de aceite usado es bombeado por P-103 hacia dos intercambiadores de calor HE-103 y HE-104, en serie con respecto al proceso. para ser calentada hasta una temperatura superior a $320{ }^{\circ} \mathrm{C}$, antes de ser alimentado al destilador flash del SKID 3 que opera a una presión de vacío menor a $13.79 \mathrm{KPa}$. Los productos obtenidos de la destilación son la base lubricante y los asfáltenos.

El circuito de aceite térmico, lo conforman principalmente por una caldera $\mathrm{H}-101$, que contiene bobinas helicoidales dobles dentro de una carcasa para el calentamiento del flujo de un aceite térmico líquido. La carcasa es calentada por un quemador y la fuente de combustible del calentador es a base de diésel.

Como aceite térmico, se utiliza uno comercial (Dowtherm A), el cual está conformado por la mezcla homogénea eutéctica de dos componentes estables: ortofenifenol y bifenilo. Ambos componentes poseen una presión de vapor similar permitiendo considerar al fluido como un solo compuesto.

\section{B. Simulación de la planta de refinación de aceites usados}

Para la cuantificación de los balances de masas y energía asociados al proceso de refinación de aceites usados y circuito térmico de calentamiento se utilizó el software PRO/II ${ }^{\circledR}$.

A los fines de disponer de la toda la información requerida por el programa de simulación, se realizó: 


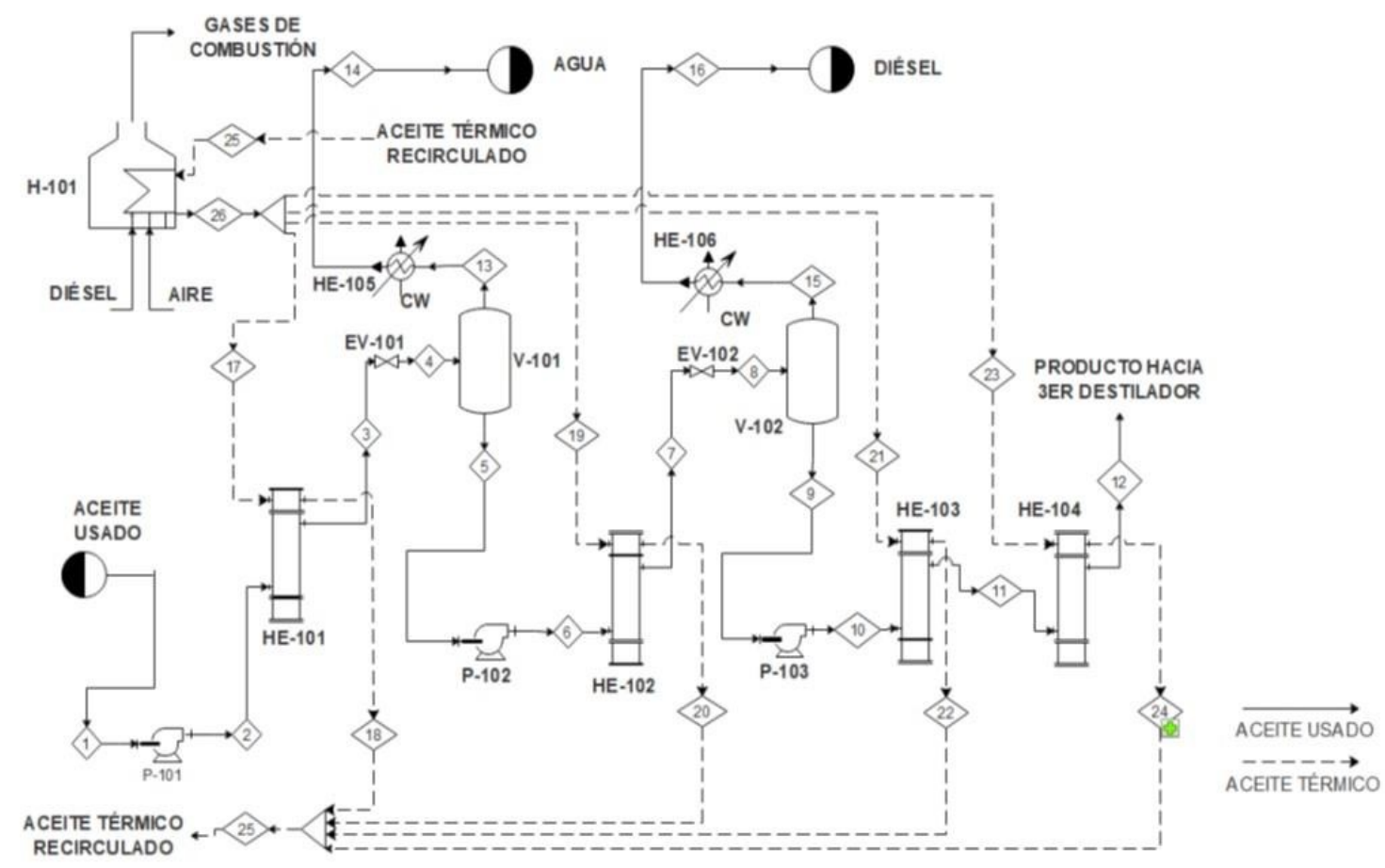

Fig. 1 Diagrama de flujo del proceso de refinación y circuito de acete térmico

$>$ Caracterización mediante pseudo-componentes del aceite usado, a partir de la destilación al vacío según procedimiento estándar D-1160, realizada en laboratorio.

$>$ Muestreo de algunas corrientes del proceso para definir las condiciones actuales, en términos de flujos, temperaturas, y disponer de especificaciones requeridas en la simulación.

La Tabla I presenta los valores promedios representativos de estos parámetros.

$>$ La selección del método termodinámico para la simulación consideró la naturaleza de las sustancias que intervienen en el proceso (moléculas apolares de hidrocarburos) y los rangos de presión y temperatura. El método elegido fue el de Peng-Robinson.

En relación a los módulos de cálculos a utilizar para la simulación, se consideró lo siguiente:

Para la simulación de la caldera, se utilizan los módulos para un reactor de conversión adiabático (RX-101) e intercambiador de calor (HX-101) con las siguientes consideraciones:

a) La reacción de combustión del diésel se simula a través de una reacción de combustión completa, con una conversión del $100 \%$, en un reactor adiabático de conversión. b) Relación molar entre el combustible y el oxígeno de 1:72 para asegurar la combustión completa del diésel.

TABLA I

VALORES PROMEDIOS DE MEDICIONES DE CORRIENTES DEL PROCESO A FINES DE CALIBRACION DE LA SIMULACION

\begin{tabular}{|c|c|c|}
\hline $\begin{array}{c}\text { Nombre o número de } \\
\text { corriente }\end{array}$ & $\begin{array}{c}\text { Flujo volumétrico } \\
(\mathrm{L} / \mathrm{h})\end{array}$ & $\begin{array}{c}\text { Temperatura } \\
\left({ }^{\circ} \mathrm{C}\right)\end{array}$ \\
\hline DIESEL & 38.10 & - \\
\hline $\begin{array}{c}\text { GASES DE } \\
\text { COMBUSTION }\end{array}$ & - & 360 \\
\hline 1 & 1088.64 & 28.7 \\
\hline 3 & - & 122.1 \\
\hline 5 & - & 88.2 \\
\hline 7 & - & 232.6 \\
\hline 9 & - & 128.3 \\
\hline 11 & - & 204.6 \\
\hline 12 & 79380.00 & 220.3 \\
\hline 25 & - & 276.09 \\
\hline 26 & & \\
\hline
\end{tabular}

c) Para la corriente de aire se especificó la composición molar de $21 \%$ de oxígeno y $79 \%$ de nitrógeno, y temperatura de $25^{\circ} \mathrm{C}$. 
d) La corriente de diésel será representada por el nhexadecano, considerando que este compuesto en mayor composición en el combustible, y su poder calorífico (PCI) es de $44.312 \mathrm{MJ} / \mathrm{kg}$ [11], cuyo valor es cercano al del PCI del diésel utilizado en el proceso, de $41.89 \mathrm{MJ} / \mathrm{kg}$. La temperatura de alimentación del diésel se supone igual a $25^{\circ} \mathrm{C}$.

Las suposiciones y especificaciones del resto de equipos para el desarrollo de la simulación se detallan en la Tabla II.

TABLA II

ESPECIFICACIONES DE MODULOS DE CALCULOS DEL PROCESO A FINES DE LA SIMULACION

\begin{tabular}{|c|c|c|c|}
\hline Equipo & Nombre & $\begin{array}{c}\text { Especificación y } \\
\text { suposiciones realizadas }\end{array}$ & Valor \\
\hline Bombas & $\begin{array}{l}\text { P-101 } \\
\text { P-102 } \\
\text { P-103 }\end{array}$ & Eficiencia & $75 \%$ \\
\hline $\begin{array}{c}\text { Intercambiador } \\
\text { del SKID } 1\end{array}$ & HE-101 & \multirow{4}{*}{$\begin{array}{l}\text { Se definen temperaturas } \\
\text { de corrientes de entrada y } \\
\text { salidas en base a } \\
\text { condiciones obtenidas de } \\
\text { muestreo, según Tabla I. }\end{array}$} & \\
\hline $\begin{array}{c}\text { Intercambiador } \\
\text { del SKID } 2\end{array}$ & HE-102 & & \\
\hline $\begin{array}{c}\text { Intercambiador } \\
1 \text { del SKID } 3\end{array}$ & HE-103 & & \\
\hline $\begin{array}{l}\text { Intercambiador } \\
2 \text { del SKID } 3\end{array}$ & HE-104 & & \\
\hline $\begin{array}{l}\text { Válvulas de } \\
\text { expansión }\end{array}$ & $\begin{array}{l}\text { GTV-101 } \\
\text { GTV-102 } \\
\text { GTV-103 }\end{array}$ & $\begin{array}{l}\text { Determinan la presión de } \\
\text { vacío en los separadores } \\
\text { flash de cada } \\
\text { opKID, } \\
\text { isoentálpica }\end{array}$ & $\leq 13.79 \mathrm{KPa}$ \\
\hline $\begin{array}{c}\text { Destilador Flash } \\
\text { del SKID } 1\end{array}$ & V-101 & \multirow{2}{*}{$\begin{array}{l}\text { Caída de presión } \\
\text { Se considera que el equipo } \\
\text { es adiabático, pues está } \\
\text { recubierto por un materia } \\
\text { aislante, evitando pérdidas } \\
\text { de calor al ambiente. }\end{array}$} & \multirow[t]{2}{*}{$0 \mathrm{KPa}$} \\
\hline $\begin{array}{c}\text { Destilador Flash } \\
\text { del SKID } 2\end{array}$ & V-102 & & \\
\hline
\end{tabular}

La temperatura del aceite térmico a la salida de cada intercambiador de calor es igual a la temperatura de retorno hacia la caldera (corriente 26).

La cuantificación de las pérdidas de energía, así como los requerimientos reales en cada etapa del proceso de refinación se determinó a partir de los resultados de la simulación, e igualmente se estimaron los flujos de aceite térmico para cada uno de los intercambiadores de calor, considerando su requerimiento energético.

\section{RESULTADOS Y DISCUSIÓN}

A. Caracterización del aceite usado, mediante una destilación al vacio según procedimiento estándar D-1160

La Tabla III presenta los resultados obtenidos de la destilación al vacío de una muestra de aceite usado proveniente de los tanques de almacenamiento de materia prima, y de donde se alimenta al proceso de refinación.
TABLA III

RESULTADOS DE DESTILACIÓN AL VACIO D-1160 PARA ACEITE USADO DE MOTOR

\begin{tabular}{|c|c|}
\hline Temperatura del líquido $\left({ }^{\circ} \mathrm{C}\right)$ & $\%$ Volumen Destilado \\
\hline 50 & 0 \\
\hline 195 & 6.5 \\
\hline 255 & 41.5 \\
\hline 305 & 84.5 \\
\hline 345 & 100 \\
\hline
\end{tabular}

Mediante la opción Assay Cut para hidrocarburos, disponible en el programa de simulación, se ingresaron los valores correspondientes a la destilación del aceite usado de la Tabla III, y a partir de esta información, el simulador genera automáticamente un grupo de pseudo-componentes y su composición, obteniéndose la composición química del aceite usado.

Para la realización de los balances de masas y energía, la composición de la alimentación de aceite usado se obtiene a partir de la composición presentada en la Tabla IV, y considerando un contenido de agua en el aceite, típicamente dado por un valor de $0.7 \%$ en volumen de agua.

TABLA IV

RESULTADOS DE DESTILACIÓN AL VACÍO D-1160 PARA ACEITE USADO DE MOTOR

\begin{tabular}{|c|c|}
\hline Pseudo-componente & Porcentaje masico $(\%)$ \\
\hline NBP-161 & 0.10 \\
\hline NBP-169 & 0.63 \\
\hline NBP-183 & 0.79 \\
\hline NBP-197 & 1.01 \\
\hline NBP-211 & 1.35 \\
\hline NBP-225 & 1.91 \\
\hline NBP-239 & 2.45 \\
\hline NBP-253 & 2.42 \\
\hline NBP-267 & 2.77 \\
\hline NBP-280 & 3.45 \\
\hline NBP-294 & 4.12 \\
\hline NBP-308 & 4.73 \\
\hline NBP-322 & 5.04 \\
\hline NBP-336 & 5.31 \\
\hline NBP-350 & 5.80 \\
\hline NBP-364 & 6.55 \\
\hline NBP-378 & 6.97 \\
\hline NBP-392 & 6.78 \\
\hline NBP-405 & 8.10 \\
\hline NBP-419 & 8.39 \\
\hline NBP-440 & 10.40 \\
\hline NBP-468 & 6.15 \\
\hline NBP-496 & 4.26 \\
\hline NBP-516 & 0.51 \\
\hline
\end{tabular}




\section{B. Rendimiento energético de la caldera}

La energía total generada por la caldera proviene exclusivamente de la combustión total del diésel alimentado, y con base a los resultados de la simulación, esta energía disponible es de $1311648.09 \mathrm{KJ} / \mathrm{h}$. Por otra parte, una fracción de esta energía es transmitida al aceite térmico, y el resto se corresponde a una energía no utilizada, y que principalmente se elimina a través de la emisión de gases de combustión a una alta temperatura, y perdidas al ambiente. La Fig. 2 muestra los resultados obtenidos en la simulación para la caldera.

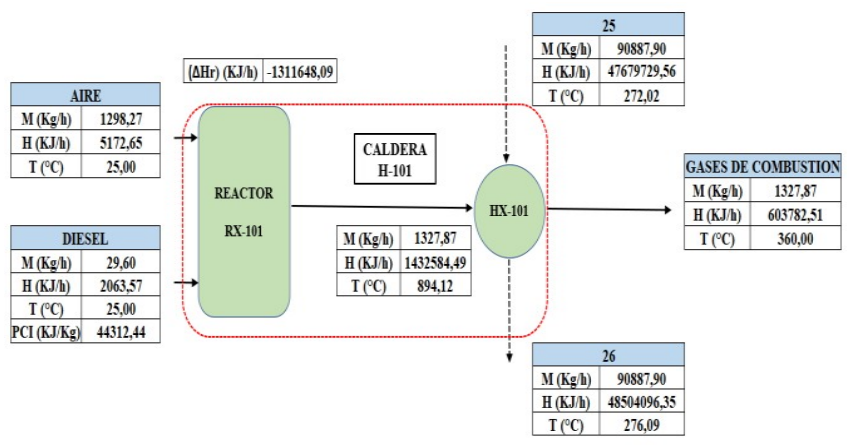

Fig. 2 Diagrama de flujo del reactor e intercambiador de calor para la simulación térmica de la caldera

El cambio de entalpia entre las corrientes 26 y 25 corresponde a la energía recibida por el aceite térmico en la caldera y es de $824366.79 \mathrm{KJ} / \mathrm{h}$, lo que representa un rendimiento de $63.2 \%$ de energía transferida, con base al total de la energía generada por la combustión de 1311648.09 $\mathrm{KJ} / \mathrm{h}$.

De un balance global de energía en el diagrama representativo para la caldera, se obtiene que las perdidas energéticas al ambiente son de $109265.00 \mathrm{KJ} / \mathrm{h}$, representando estas un $8.33 \%$.

\section{Rendimiento energético en el proceso de refinación}

De la energía disponible en el aceite térmico, igualmente una parte de esta energía es utilizada en las diferentes etapas de separación del proceso de refinación, así como una parte de esta energía se pierde al ambiente.

La cuantificación del consumo energético se realizó considerando tres etapas principales definidas como SKID 1, SKID 2 y SKID 3. La Fig. 3 presenta el diagrama esquemático de la etapa SKID 1, y de donde se determina, a partir del balance energético obtenido por la simulación, los siguientes resultados.

A las condiciones de operación actuales, del balance energético entre salidas y entradas, indica unas pérdidas al ambiente de $68622.68 \mathrm{KJ} / \mathrm{h}$, lo que representa un $0.5 \%$ de la energía alimentada a la etapa a través de las corrientes de aceite térmico (17), y aceite usado (2). Adicionalmente, una evaluación energética en cada equipo de la sección, indica que estas pérdidas son generadas principalmente en el separador flash V-101.

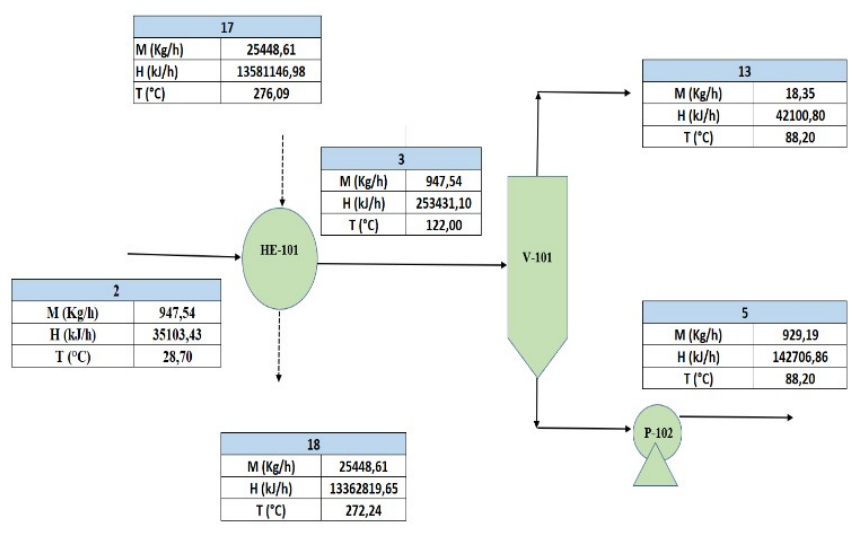

Fig. 3 Diagrama esquemático de la etapa SKID 1 del proceso de refinación y circuito de acete térmico

En relación a la etapa SKID 2, cuyo diagrama se presenta en la Fig. 4, a partir del balance energético global en la etapa, resultan unas pérdidas al ambiente de $266550.10 \mathrm{KJ} / \mathrm{h}$, equivalente a un $1.21 \%$ de la energía alimentada a través de las corrientes de aceite térmico (19) y aceite usado proveniente del SKID 1 (6).

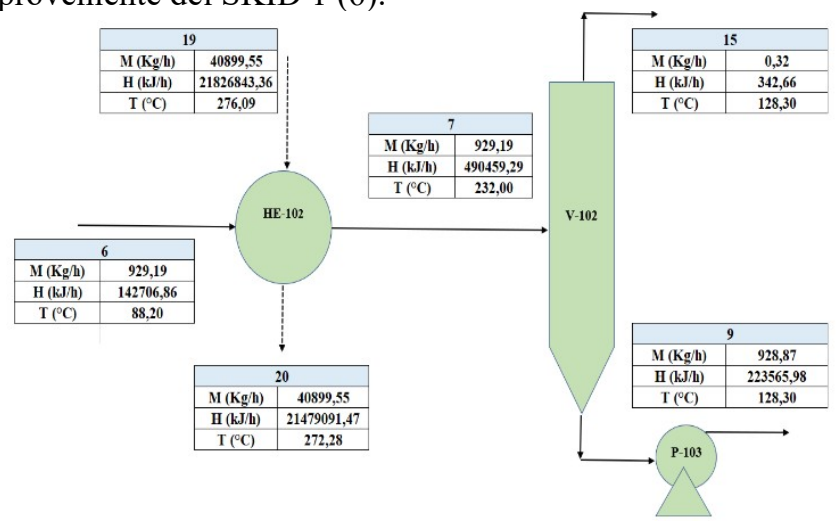

Fig. 4 Diagrama esquemático de la etapa SKID 2 del proceso de refinación y circuito de acete térmico

En esta etapa las perdidas energéticas resultan mayores a las determinadas en la etapa previa, debido a las mayores temperaturas a las cuales están los equipos en esta sección del proceso. Igualmente, a lo observado anteriormente, las mayores pérdidas provienen del equipo separador $\mathrm{V}-102$.

La Fig. 5 muestra el diagrama esquemático de los intercambiadores de calor utilizados en el calentamiento del aceite usado a destilarse en el SKID 3, separador donde finamente se recupera la base lubricante presente en el aceite usado.

A partir del balance energético global en la etapa, resultan unas pérdidas al ambiente relativamente bajas, considerando el aislamiento de los intercambiadores, así como un área externa superficial pequeña en estos intercambiadores de calor. 


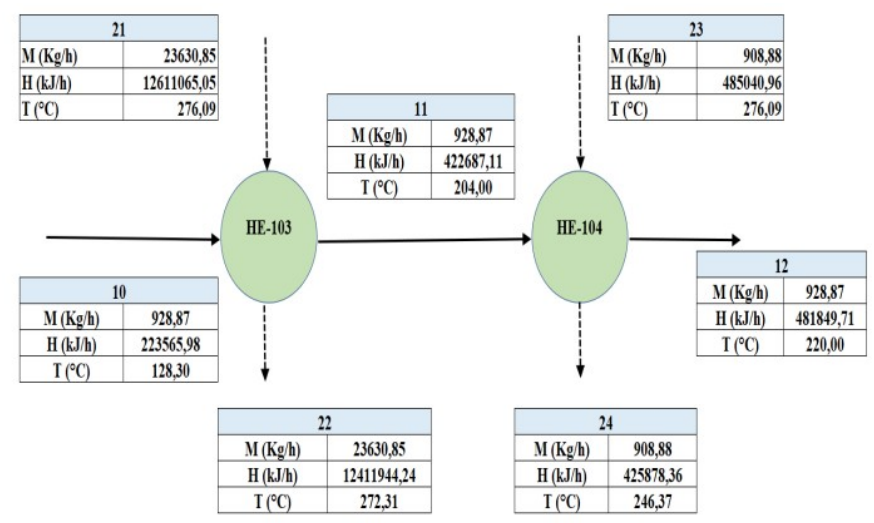

Fig. 5 Diagrama esquemático de la etapa SKID 3 del proceso de refinación y circuito de acete térmico

\section{Distribución energética en las etapas del proceso de refinación}

Un aspecto importante a determinar en el proceso de refinación, es la distribución energética o fracción de energía requerida en las etapas definidas como parte del proceso de refinación.

Del total de los $824366.79 \mathrm{KJ} / \mathrm{h}$ recibidos por el aceite térmico en la caldera, a partir de los cambios de entalpias del aceite térmico una vez pasa a través de los cuatro intercambiadores da calor, se obtienen los resultados mostrados en la Tabla V.

TABLA V

DISTRIBUCION PORCENTUAL DE CONSUMOS ENERGETICOS POR ETAPAS EN PROCESO DE REFINACION DE ACEITES USADOS

\begin{tabular}{|c|c|c|}
\hline $\begin{array}{c}\text { Intercambiador } \\
\text { de calor }\end{array}$ & $\begin{array}{c}\text { Cambio de entalpia } \\
(\mathrm{KJ} / \mathrm{h})\end{array}$ & $\begin{array}{c}\text { Porcentaje del total } \\
\text { disponible }(\%)\end{array}$ \\
\hline HE-101 & 218327.33 & 26.48 \\
\hline HE-102 & 347751.89 & 42.18 \\
\hline HE-103 & 199120.81 & 24.15 \\
\hline HE-104 & 59162.60 & 7.18 \\
\hline
\end{tabular}

Los resultados indican que el mayor requerimiento lo tiene en intercambiador de calor HE-102, puesto que es allí donde se requiere un mayor incremento en la temperatura del aceite usado, desde $88^{\circ} \mathrm{C}$ hasta $232^{\circ} \mathrm{C}$.

Adicionalmente, se observa un porcentaje mayor a lo esperado para el intercambiador HE-101, lo que explica una temperatura más elevada de la deseada de $90{ }^{\circ} \mathrm{C}$, igual a 122 ${ }^{\circ} \mathrm{C}$.

Los resultados indican que los intercambiadores HE-103 y HE-104, donde se utiliza cerca de un $32 \%$ de la energía disponible, no tienen la capacidad suficiente para incrementar la temperatura del aceite usado antes de alimentarse al separador flash, hasta $320{ }^{\circ} \mathrm{C}$, temperatura definida como condición de alimentación. A las condiciones actuales de operación esta temperatura está en $220{ }^{\circ} \mathrm{C}$, por lo que se recomienda una evaluación del diseño de estos dos intercambiadores de calor.

\section{E. Evaluación de potencial energético recuperable del circuito de aceite térmico}

Los resultados indican que, de la energía total generada por efectos de la combustión de diésel, un $63.2 \%$ de esta energía es transferida al aceite térmico, y que las perdidas energéticas al ambiente son de un $8.33 \%$, por lo que hay una energía residual equivalente a un $28.47 \%$, es decir 378016.30 $\mathrm{KJ} / \mathrm{h}$, y asociada a los gases de combustión emitidos a altas temperaturas.

Se evalúa la instalación de un economizador, donde se recupera parte de esta energía, mediante el enfriamiento de los gases calientes hasta una temperatura dada, y en casa caso cuantificar a partir de la energía recuperada un equivalente expresado en cantidad de diésel a utilizar, considerando una eficiencia de recuperación de un $75 \%$ para el economizador.

La tabla VI presenta, la energía disponible, en función de la temperatura de salida de los gases de combustión, una vez tratados en el economizador, y el flujo masico equivalente en diésel, respecto al utilizado actualmente de $29.60 \mathrm{Kg} / \mathrm{h}$, y con un PCI de $44312 \mathrm{KJ} / \mathrm{Kg}$. Se indica el porcentaje de este flujo de diésel, con base al flujo actualmente utilizado en el proceso de refinación.

TABLA VI

DISPONIBILIDAD ENERGETICA POR ENFRIAMIENTO DE GASES DE COMBUSTION EN FUNCION DE LA TEMPERATURA DE EMISION

\begin{tabular}{|c|c|c|c|}
\hline $\begin{array}{c}\text { Temperatura } \\
\text { salida de } \\
\text { gases }\left({ }^{\circ} \mathrm{C}\right)\end{array}$ & $\begin{array}{c}\text { Energía } \\
\text { disponible en el } \\
\text { economizador } \\
(\mathrm{KJ} / \mathrm{h})\end{array}$ & $\begin{array}{c}\text { Flujo } \\
\text { equivalente } \\
\text { en diésel } \\
(\mathrm{Kg} / \mathrm{h})\end{array}$ & $\begin{array}{c}\text { Porcentaje de } \\
\text { diésel en base al } \\
\text { actual de } 29.60 \\
\mathrm{Kg} / \mathrm{h}\end{array}$ \\
\hline 300 & 87120 & 1.97 & 6.64 \\
\hline 250 & 159000 & 3.59 & 12.12 \\
\hline 200 & 230000 & 5.19 & 17.54 \\
\hline 150 & 300000 & 6.77 & 22.87 \\
\hline
\end{tabular}

Los resultados muestran la posibilidad de recuperar un equivalente al diésel actualmente utilizado, de hasta un $22.8 \%$, en caso de implementar un sistema de recuperación de la energía disponible en los gases calientes de combustión.

\section{CONCLUSIONES}

El estudio realizado en una refinería en operación, con una capacidad de procesamiento de $280 \mathrm{GPH}$ de aceite usado de motor, indica que, en la caldera para calentamiento de aceite térmico, presenta una eficiencia del $63.2 \%$, respecto al total de energía generada por efectos de una combustión total y completa de diésel. Igualmente se determinó una pérdida de energía al ambiente en la caldera de un $8.33 \%$, y se tiene una disponibilidad de recuperación de energía, proveniente de los gases calientes de un $28.47 \%$. 
De la energía suministrada al aceite térmico, fuente de calentamiento de las diferentes secciones del proceso de rerefinación, se determinó que, en la etapa de eliminación de agua e hidrocarburos ligeros, se utiliza un $26.48 \%$ de la energía total, en la etapa de eliminación de diésel se utiliza un $42.18 \%$, mientras que para el precalentamiento de aceite usado previa a la destilación de la base lubricante se utiliza un $31.33 \%$. En las etapas de refinación existen unas pérdidas al ambiente a través de intercambiadores de calor, tuberías y evaporadores equivalentes al $1.2 \%$ de la energía total suministrada al aceite térmico.

En el caso de realizar una recuperación de $300000 \mathrm{KJ} / \mathrm{h}$ a partir de la energía disponible en los gases de combustión, ello representaría una cantidad equivalente a un $22.87 \%$ del diésel utilizado actualmente en el proceso.

\section{REFERENCES}

K. Boadu, O. Joel, D. Essumang, and B. Evbuomwan, "A Review of Methods for Removal of Contaminants in Used Lubricating Oil," Chem. Sci. Int. J., pp. 1-11, Apr. 2019, doi: 10.9734/CSJI/2019/v26i430101.

[2] J. Santos, "Recovery of Used Lubricating Oils-A Brief Review," Prog. Petrochemical Sci., vol. 1, Apr. 2018, doi: 10.31031/PPS.2018.01.000516.

[3] C. Sánchez-Alvarracín, J. Criollo-Bravo, D. Albuja-Arias, F. García-Ávila, and M. R. Pelaez-Samaniego, "Characterization of Used Lubricant Oil in a Latin-American Medium-Size City and Analysis of Options for Its Regeneration Characterization of Used Lubricant Oil in a Latin-American Medium-Size City and Analysis of Options for Its Regeneration," Recycling, vol. 6, Feb. 2021, doi: 10.3390/recycling6010010

[4] J. A. Botas, J. Moreno, J. J. Espada, D. P. Serrano, and J. Dufour, "Recycling of used lubricating oil: Evaluation of environmental and energy performance by LCA," Resour. Conserv. Recycl., vol. 125, no. July, pp. 315-323, 2017, doi: 10.1016/j.resconrec.2017.07.010.

[5] N. Zgheib and H. Takache, "Recycling of used lubricating oil by solvent extraction: experimental results, Aspen Plus simulation and feasibility study," Clean Technol. Environ. Policy, vol. 23 Jan. 2021, doi: 10.1007/s10098-020-01893-0.

[6] L. M. Corredor-Rojas, A. Uribe-Rodríguez, and J. C. CobosGómez, "Monitoring and evaluation of energy use in oil treatmen facilities," $D Y N A$, vol. 83, no. 198, pp. 142-147, 2016, doi: 10.15446/dyna.v83n197.49600.

[7] E. Negri et al., "Improving Energy Efficiency In Upstream Facilities ."Mar. 23, 2011.

[8] F. Zhao, Y. Fan, and S. Zhang, "Assessment of efficiency improvement and emission mitigation potentials in China's petroleum refining industry," J. Clean. Prod., vol. 280, p. 124482 , Jan. 2021, doi: 10.1016/J.JCLEPRO.2020.124482.

[9] A. Espinoza and S. Macias, "Evaluación energética del sistema de calentamiento de aceite térmico en una refinería de aceite usado de motor.," Escuela Superior Politécnica del Litoral-Ecuador, 2020.

[10] T. Humpreys and J. Nichols, "ARR-PATENT-2018PROCESS.pdf," US 9,957,445 B2, 2018.

[11] A. Pérez-Sánchez, G. Montero-Alpírez, R. Ayala-Bautista, M. A Coronado-Ortega, C. García-González, and H. E. Campbell-
Ramírez, "Simulación en Aspen de la combustión de mezclas diesel-biodiesel," Ing. Investig. y Tecnol., vol. 16, no. 1, pp. 8392, 2015, Accessed: Jul. 20, 2021. [Online]. Available: http://www.scielo.org.mx/scielo.php?script=sci_arttext\&pid=S14 05-77432015000100008\&lng=es\&nrm=iso\&tlng=es. 